\title{
Signal intensity change on unenhanced T1-weighted images in dentate nucleus following gadobenate dimeglumine in patients with and without previous multiple administrations of gadodiamide
}

\author{
Joana Ramalho ${ }^{1,2} \cdot$ Richard C Semelka $^{1} \cdot$ Mamdoh AlObaidy $^{1,3} \cdot$ Miguel Ramalho $^{1,4}$. \\ Renato H Nunes $^{1,5}$ - Mauricio Castillo ${ }^{1}$
}

Received: 16 September 2015 /Revised: 24 January 2016 / Accepted: 3 February 2016/Published online: 24 February 2016

(C) European Society of Radiology 2016

\begin{abstract}
Objectives To evaluate the impact of previous administration of gadodiamide and neural tissue gadolinium deposition in patients who received gadobenate dimeglumine.

Methods Our population included 62 patients who underwent at least three administrations of gadobenate dimeglumine, plus an additional contrast-enhanced last MRI for reference, divided into two groups: group 1, patients who in addition to gadobenate dimeglumine administrations had prior exposure
\end{abstract}

Miguel Ramalho

miguel-ramalho@netcabo.pt

Joana Ramalho

joana-ramalho@netcabo.pt

Richard C Semelka

richsem@med.unc.edu

Mamdoh AlObaidy

mamdoh.alobaidy@yahoo.com

Renato H Nunes

renatohn@hotmail.com

Mauricio Castillo

mauricio_castillo@med.unc.edu

1 University of North Carolina Hospital, Chapel Hill, NC, United States

2 Centro Hospitalar de Lisboa Central, Lisbon, Portugal

3 King Faisal Specialist Hospital \& Research Center, Riyadh, Saudi Arabia

4 Hospital Garcia de Orta, Almada, Portugal

5 Santa Casa de Misericórdia de São Paulo, São Paulo, Brazil to multiple doses of gadodiamide; group 2, patients without previous exposure to other gadolinium-based contrast agent (GBCAs). Quantitative analysis was performed on the first and last gadobenate dimeglumine MRIs in both groups. Dentate nucleus-to-middle cerebellar peduncle signal intensity ratios (DN/MCP) and relative change (RC) in signal over time were calculated and compared between groups using generalized additive model.

Results Group 1 showed significant increase in baseline and follow-up DN/MCP compared to group $2(p<0.0001)$. The RC DN/MCP showed a non-statistically significant trend towards an increase in patients who underwent previous gadodiamide $(p=0.0735)$.

Conclusion There is increased T1 signal change over time in patients who underwent gadobenate dimeglumine and had received prior gadodiamide compared to those without known exposure to previous gadodiamide. A potentiating effect from prior gadodiamide on subsequent administered gadobenate dimeglumine may occur.

Key Points

- Neural gadolinium deposition is associated with multiple administrations of less stable GBCAs.

- Less stable GBCA effect on subsequent more stable GBCA administrations is undetermined.

- Significant increase of DN/MCP was seen in patients with previous gadodiamide exposure.

- RC DN/MCP showed a non-significant increase in patients who received previous gadodiamide.

- Potentiating effects from prior gadodiamide on subsequent administered gadobenate dimeglumine may occur.

Keywords Gadolinium deposition · Gadodiamide . Gadobenate dimeglumine $\cdot$ Neural tissue $\cdot$ Dentate nucleus 


$\begin{array}{ll}\text { Abbreviations } \\ \text { DN } & \text { dentate nucleus } \\ \text { GBCA } & \text { gadolinium-based contrast agent } \\ \text { MCP } & \text { middle cerebellar peduncle } \\ \text { RC } & \text { relative change } \\ \text { SI } & \text { signal intensity }\end{array}$

\section{Introduction}

Over the last 2 years, reports have been published describing progressive increase in signal intensity in the globus pallidi (GP) and/or dentate nuclei (DN) on unenhanced T1-weighted images in patients with normal renal function who were exposed to multiple administrations of different gadoliniumbased contrast agents (GBCAs), such as gadodiamide (Omniscan ${ }^{\circledR}$, GE Healthcare) [1-4] and gadopentetate dimeglumine (Magnevist ${ }^{\circledR}$, Bayer Healthcare Pharmaceuticals) [1, 5-7].Recently, human [8,9] and animal $[10,11]$ histopathology studies have shown a dose-dependent relationship between intravenous GBCAs administration and subsequent neural deposition of gadodiamide (Omniscan ${ }^{\circledR}$ ) $[8,10,11]$, gadopentetate dimeglumine (Magnevist $\left.{ }^{\circledR}\right)[9,11]$ and gadobenate dimeglumine (MultiHance ${ }^{\circledR}$, Bracco Diagnostics) [11]. Those studies $[8,10,11]$ confirmed a positive correlation between T1-weighted signal intensity and gadolinium tissue deposition.

Other more stable macrocyclic GBCAs, such as gadoteridol (ProHance ${ }^{\circledR}$, Bracco Diagnostics) [5], gadoterate meglumine (Dotarem ${ }^{\circledR}$, Guerbet) $[6,10,11]$ and gadobutrol (Gadavist ${ }^{\circledR}$, Bayer Healthcare Pharmaceuticals) [7, 12] have not been associated with substantial brain MRI signal intensity changes. Gadobenate dimeglumine (MultiHance ${ }^{\circledR}$ ) an agent of intermediate stability was associated with fewer MRI changes compared with the linear gadodiamide [4]. Recently, Weberling et al. [13] suggested that this agent releases less gadolinium than gadopentetate dimeglumine (Magnevist ${ }^{\circledR}$ ) but more than gadoterate meglumine (Dotarem $\left.{ }^{\circledR}\right)$. An animal study by Robert et al. [11] corroborated this finding. However, a lack of appreciable MRI findings does not exclude human neural tissues deposition even with the more stable GBCAs because a lesser deposition may not be observable on clinical MRI studies.

In clinical practice, patients may undergo MRI with different gadolinium chelates for a variety of reasons including being studied at different facilities and changes in MRI contrast agents at the same imaging centre. An explanation for these changes is the general shift from less stable GBCAs to more stable GBCAs. To the best of our knowledge, the effect of previous administration of different GBCAs in neural tissue deposition has not been evaluated.

Our purpose was to evaluate the effect of previous administration of gadodiamide, a linear non-ionic GBCA associated with significant neural tissue deposition, in patients who subsequently received gadobenate dimeglumine, a more stable linear ionic GBCA associated with minimal tissue deposition [14]. To achieve our goal, we compared T1-weighted changes in patients after multiple MRI studies with gadobenate dimeglumine and without previous exposure to other GBCAs to patients who underwent multiple studies with gadobenate dimeglumine and previously received multiple doses of gadodiamide.

\section{Materials and methods}

Institutional review board (IRB) approval with signature waiver was obtained for this Health Insurance Portability and Accountability Act (HIPAA)-compliant retrospective longitudinal observational cohort study.

\section{Study population}

In our databases, we identified 200 consecutive patients who underwent at least three GBCA administrations, plus an additional MRI study as reference. At our institution we used gadodiamide as the sole GBCA for all enhanced MRI studies performed before December 2006 irrespective of the patient's renal function. From December 2006 to June 2007, patients with normal renal function still received gadodiamide and after June 2007 all adult patients received gadobenate dimeglumine and the use of gadodiamide was discontinued.

Patients who underwent GBCA administration outside of our institution or who underwent MRI with a GBCA other than gadodiamide or gadobenate dimeglumine $(n=8)$ were excluded. Patients who received only gadodiamide were also excluded $(n=5)$. To avoid confounding factors, patients with a history of targeted or whole-brain radiation therapy $(n=69)$ [15], those with multiple sclerosis $(n=23)$ [16] and those with unknown diagnosis $(n=2)$ were also excluded. Other exclusion criteria were the absence of unenhanced spin-echo T1weighted MR images $(n=22)$, unsatisfactory images due to artefacts and/or brain lesions involving the $\mathrm{DN}(n=7)$ and examinations performed at field strengths other than 1.5 Tesla (T) $(n=2)$. Our final population included 62 patients (Fig. 1).

Among those 62 patients who underwent multiple gadobenate dimeglumine studies, 18 had previous administration of gadodiamide and those constituted group 1 . The remaining 44 patients who received only gadobenate dimeglumine constituted group 2 . For both groups, the minimum number of studies using gadobenate dimeglumine for a given patient was three plus a fourth examination used as a reference for measuring the follow-up signal. For each patient, the number of gadolinium-enhanced MRI examinations performed with gadodiamide and gadobenate dimeglumine was 
200 patients with $\geq 3$ contrast-enhanced MRI examinations, plus an additional MRI examination $\left(\right.$ Exam $\left._{\text {Final }}\right)$ used as a reference.

\section{GBCA Related}

- GBCA administration outside our institution or with a GBCA other than gadodiamide or gadobenate dimeglumine $(n=8)$

- Usage of gadodiamide alone $(n=5)$.

\section{Clinically Related}

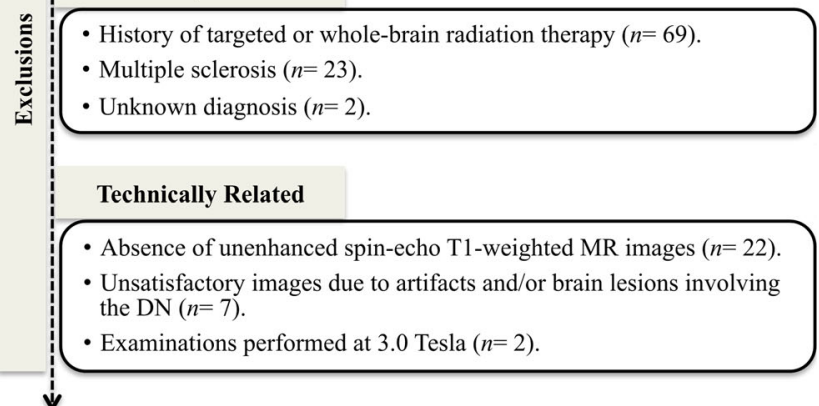

62 patients with $\geq 3$ contrast-enhanced MRI examinations, plus an additional MRI examination (Exam Final $)$ used as a reference.

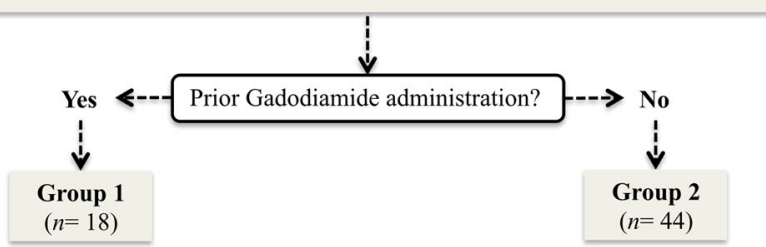

Fig. 1 Study flowchart of included and excluded patients

recorded. Imaging analysis was performed only for gadobenate dimeglumine-enhanced MRIs. The first brain contrast-enhanced MRI examination performed with

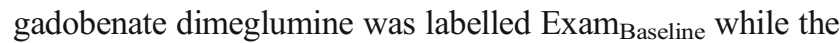
last contrast-enhanced MRI examination was labelled Exam $_{\text {Final }}$ (Fig. 2).

Age, gender, diagnosis, liver function tests and estimated glomerular filtration rate (eGFR) were recorded for all patients. Liver function was assessed by serum concentrations of aspartate aminotransferase, alanine aminotransferase, total bilirubin, or gamma-glutamyl transpeptidase. Normal liver function was considered if these laboratory measurements were within the normal limits. Renal function was assessed by means of the eGFR. Normal renal function was considered when eGFR $\geq 60 \mathrm{~mL} / \mathrm{min} / 1.73 \mathrm{~m}^{2}$. The patients' demographics and examinations are summarised for each group in Table 1. The number of gadolinium administrations for each GBCA are shown for each group in Table 2.

\section{Imaging protocol}

Brain MRI was performed on a 1.5-T system (Magnetom $®$ Avanto; Siemens, Erlangen, Germany) with a dedicated 12element matrix head coil. Imaging protocols varied according to the patient's clinical indication but all included fast spinecho (SE) T1-weighted images with the following parameters: repetition time (TR), $623 \mathrm{~ms}$; echo time (TE), $13 \mathrm{~ms}$; echo train length, 1; section thickness, $5 \mathrm{~mm}$; spacing, $1 \mathrm{~mm}$; matrix size, $256 \times 256$; and field-of-view, $165 \times 220$. A standard dose of $0.1 \mathrm{mmol}$ of gadodiamide or gadobenate dimeglumine

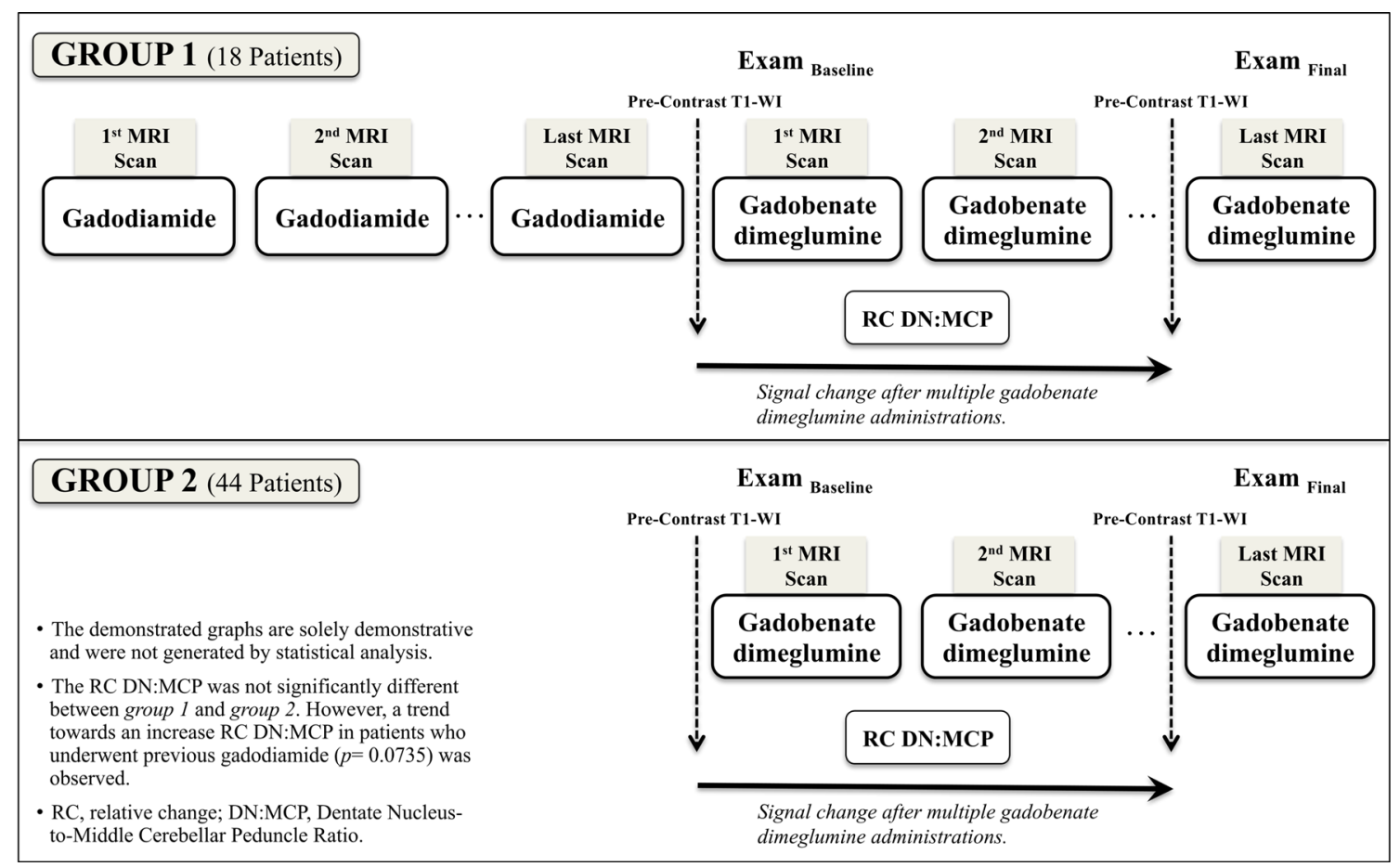

Fig. 2 Schematic representation of the study design 
Table 1 Patients' demographics and examinations description

\begin{tabular}{lll}
\hline & Group 1 & Group 2 \\
\hline Number of patients & 18 & 44 \\
Age & $56.5 \pm 13.9(29-84$ years $)$ & $60.4 \pm 14.2(29-86$ years $)$ \\
Gender & 16 female, 2 male & 23 female, 21 male \\
Number of MRIs with gadodiamide & $5.9 \pm 2.7(3-11)$ & NA \\
Number of MRIs with gadobenate dimeglumine & $5.5 \pm 1.9(3-10)$ & $4.5 \pm 2.0(3-11)$ \\
Interval (MRI gadobenate dimeglumine) & $1762.4 \pm 433.5(801-2315$ days) & $1089.9 \pm 592.3(94-2633$ days $)$ \\
Diagnosis & Meningioma $(n=8)$ & Pituitary lesions $(n=15)$ \\
& Pituitary lesions $(n=4)$ & Stroke $(n=11)$ \\
& Stroke $(n=2)$ & Meningioma $(n=6)$ \\
& PRES $(n=1)$ & Breast cancer without brain metastases $(n=3)$ \\
& Intracranial aneurysm $(n=1)$ & Vestibular schwannoma $(n=2)$ \\
& Clivus chordoma $(n=1)$ & Clivus chordoma $(n=1)$ \\
& Spinal haemangioblastoma $(n=1)$ & Trigeminal mass $(n=1)$ \\
& & CSF leak $(n=1)$ \\
& & Intraventricular mass $(n=1)$ \\
& & Trauma $(n=1)$ \\
& & Acute haematoma $(n=1)$ \\
& & Epidermoid $(n=1)$
\end{tabular}

Gadodiamide, Omniscan ${ }^{\circledR}$; gadobenate dimeglumine, MultiHance ${ }^{\circledR}$

NA not applicable, PRES posterior reversible encephalopathy syndrome, CSF cerebrospinal fluid

Table 2 Number of doses administered for patients in both groups

\begin{tabular}{lllll}
\hline Group 1 & \multicolumn{3}{l}{ Group 2 } \\
\hline $\begin{array}{l}\text { Patient } \\
\text { ID }^{\mathrm{a}}\end{array}$ & Gadodiamide & $\begin{array}{c}\text { Gadobenate } \\
\text { dimeglumine }\end{array}$ & Frequency & Gadobenate \\
1 & 8 & 4 & 17 & 3 \\
2 & 9 & 8 & 12 & 4 \\
3 & 6 & 5 & 7 & 5 \\
4 & 11 & 5 & 3 & 6 \\
5 & 5 & 4 & 2 & 7 \\
6 & 8 & 10 & 2 & 10 \\
7 & 3 & 7 & 1 & 11 \\
8 & 3 & 5 & & \\
9 & 7 & 6 & & \\
10 & 3 & 7 & & \\
11 & 6 & 4 & & \\
12 & 3 & 8 & & \\
13 & 4 & 4 & & \\
14 & 6 & 4 & & \\
15 & 4 & 3 & & \\
16 & 3 & 6 & & \\
17 & 6 & 4 & & \\
18 & 11 & 5 & & \\
\hline
\end{tabular}

Gadodiamide, Omniscan ${ }^{\circledR}$; gadobenate dimeglumine, MultiHance ${ }^{\circledR}$

${ }^{\text {a }}$ Refers to individual patients' ID numbers

${ }^{\mathrm{b}}$ Frequency represents the number of patients who received the stated number of gadobenate dimeglumine administrations in group 2, which did not have a prior exposure to gadodiamide per kilogram of body weight was administered intravenously using a power injector (Medrad, Pittsburgh, PA) at a rate of $1.5-2.0 \mathrm{~mL} / \mathrm{s}$ followed by a $20-\mathrm{mL}$ saline flush bolus at the same rate.

\section{Imaging analysis}

Images were independently evaluated on a dedicated workstation (IMPAX® v.6, Agfa Healthcare, Mortsel, Belgium) by two radiologists ( $J R$ and $R H N$ ). The sequences were randomly loaded into the workstation and all patient information was removed by a coauthor who did not take part in the actual image evaluation. Quantitative analysis was performed on gadobenate dimeglumine contrast-enhanced MRIs. A region-of-interest (ROI)-based approach was applied for both the first $\left(\right.$ Exam $\left._{\text {Baseline }}\right)$ and last (Exam Final $\left._{1}\right)$ unenhanced T1weighted images. Oval ROIs were manually placed on the $\mathrm{DN}$ and middle cerebral peduncles (MCPs) including as much of the anatomic structures as possible while avoiding lesions, blood vessels and/or artefacts. To ascertain a correct placement of the ROI, T2-weighted or diffusion-weighted images (DWI) were used to increase confidence in identifying the DN. Whenever possible, DN and MCP measurements were averaged for right and left sides and for both readers.

The DN-to-MCP signal intensity ratio (DN/MCP) was calculated by dividing the mean signal intensity of the $\mathrm{DN}$ by that of the MCP. This ratio was determined for the baseline and final MRI examinations performed with gadobenate dimeglumine in all patients in both groups. 
The relative change $(\mathrm{RC})$ of $\mathrm{DN} / \mathrm{MCP}$ was then calculated for all patients using the first MRI measurement as reference according to the following equation where Final indicates the last contrast-enhanced MRI study and Baseline indicates the first contrast-enhanced MRI study in the same patient [2]:

$R C_{(D N / M C P)}=\frac{D N / M C P_{\text {Final }}-D N / M C P_{\text {Baseline }}}{D N / M C P_{\text {Baseline }}}$

\section{Statistical analysis}

$\mathrm{R}$, a language and environment for statistical computing ( $\mathrm{R}$ Core Team, Vienna, Austria), was used for all statistical computing [17]. The Mixed GAM Computation Vehicle "mgcv" package with GCV/AIC/REML Smoothness [18] was used to apply the generalized additive model (GAM). Statistical significance was defined as a $p$ value less than 0.05 .

Interobserver correlation agreement between the two readers' ROI measurements for each structure was tested using Lin's concordance correlation coefficient [19]. Intraobserver agreement between right and left measurements was also tested with Lin's concordance correlation coefficient. The strength of correlation was considered almost perfect when it was greater than 0.99 , substantial when it was 0.95 0.99 , moderate when it was $0.90-0.95$ and poor when it was less than 0.90

The DN/MCP ratios as well as RC in ratios were individually evaluated as dependent variables by using GAM. The GAM function is a non-parametric regression analysis evaluated by using the GAM computation vehicle with generalized cross validation, the Akaike information criterion and restricted maximum likelihood (GCV/AIC/REML) smoothness estimation function. The model for both ratios and $\mathrm{RC}$ in ratios was defined as follows:

$s($ number of MRIs $)+s($ age $)+s($ interval $)+$ gender

+ prior exposure to gadodiamide

The function $s$ was defined as a smoothing spline function with penalized regression splines to specify that the subsequent linear predictors depend on smooth functions. Smoothing parameters were calculated as minimalized generalized cross validation.

\section{Results}

All patients included in our study had normal liver and renal functions. The left DN and MCP were excluded in one patient from group 1 and the right $\mathrm{DN}$ and MCP were excluded in one patient from group 2 (both for baseline and final MRI examinations) because of image artefacts. In these two patients, ratios were calculated on the basis of the contralateral values alone without averaging.

A total of 140 ROIs were drawn for group 1 and 348 were for group 2. Interobserver agreement was almost perfect for all four structures evaluated (0.99). Intraobserver agreement was substantial to almost perfect for both readers. The Lin's concordance correlation coefficient was 0.99 for DN and MCP for reader 1 , and 0.98 for DN and 0.99 for MCP for reader 2 .

Group 1 included 18 patients ( 16 female, 2 male; mean age 56.5 years \pm 13.9 , range $29-84$ ). The number of administered doses of gadodiamide ranged from 3 to 11 (mean 5.9 doses \pm 2.7 ). The number of administered doses of gadobenate dimeglumine ranged from 3 to 10 (mean 5.5 doses \pm 1.9 ) and the interval between the baseline and last examinations ranged from 801 to 2315 days (mean 1762.4 days \pm 433.5 ).

Group 2 included 44 patients ( 23 female, 21 male; mean age 60.4 years \pm 14.2 , range $29-86$ ). The number of administered doses of gadobenate dimeglumine ranged from 3 to 11 (mean 4.5 doses \pm 2.0 ) and the intervals between the baseline and final examinations ranged from 94 to 2633 days (mean 1089.9 days \pm 592.3).

Detailed results of the GAM data analysis are shown in Table 3. Patients who received gadodiamide before gadobenate dimeglumine showed a significant increase in baseline DN/MCP $(p<0.0001)$ compared with those without previous administration of gadodiamide.

The RC DN/MCP was not significantly different between group 1 and group 2 . However, a non-statistically significant trend towards an increase $\mathrm{RC} \mathrm{DN} / \mathrm{MCP}$ in patients who underwent previous gadodiamide $(p=0.0735)$ was observed. (Figs. 3, 4, and 5).

Gender, age and intervals did not have significant influence on RC DN/MCP.

\section{Discussion}

Our results show that DN/MCP for baseline and final examinations were significantly increased in patients who underwent previous exposure to gadodiamide compared to those who underwent studies using only gadobenate dimeglumine only $(p<0.0001)$.

Prior studies [1-4] described a progressive increase in T1 signal in the dentate nuclei associated with gadolinium deposition after multiple administrations of gadodiamide. Additionally, a recent animal study [10] employing multiple administrations of gadodiamide showed that the signal hyperintensity in the DN did not resolve during a 5-week contrast-free period, suggesting persistence of this phenomenon after the last administration of the contrast with no washout effect over a period of weeks. These observations are in accordance with our results. 
Table 3 Results of non-parametric regression models

\begin{tabular}{|c|c|c|c|c|c|c|}
\hline Signal intensity ratio & Parameter & $\begin{array}{l}\text { Estimated value } \\
\text { of parametric coefficients }\end{array}$ & Standard error & $\begin{array}{l}\text { Estimated degrees } \\
\text { of freedom }\end{array}$ & $\begin{array}{l}\text { Reference degrees } \\
\text { of freedom }\end{array}$ & $p$ value \\
\hline \multirow[t]{5}{*}{$\mathrm{DN} / \mathrm{MCP}$} & Gender & 0.007427 & 0.010457 & - & - & 0.479 \\
\hline & Previous & 0.050625 & 0.011527 & - & - & $<0.0001$ \\
\hline & Age & - & - & 2.313 & 2.894 & 0.0385 \\
\hline & MRIs & - & - & 1 & 1 & 0.4455 \\
\hline & Interval & - & - & 1.508 & 1.843 & 0.206 \\
\hline \multirow[t]{5}{*}{ Relative change DN/MCP } & Gender & 0.003634 & 0.015788 & - & - & 0.8188 \\
\hline & Previous & 0.035266 & 0.01933 & - & - & 0.0735 \\
\hline & Age & - & - & 1.157 & 1.297 & 0.52 \\
\hline & MRIs & - & - & 1.441 & 1.687 & 0.069 \\
\hline & Interval & - & - & 1 & 1 & 0.483 \\
\hline
\end{tabular}

Data represent analysis of signal intensity ratios using the GAM function

$D N / M C P$ dentate nuclei-to-middle cerebellar peduncle ratio, Previous previous administration of gadodiamide, MRIs number of MRIs performed, GAM generalized additive models

An investigation by Ramalho et al. [4] compared the accumulation of gadodiamide and gadobenate dimeglumine in the GP and DN. They showed a significant increased signal intensity in the GP and DN associated with multiple gadodiamide-enhanced studies but not with gadobenate dimeglumine-enhanced studies. Rate-of-change data indirectly suggested that gadolinium deposition occurred in the DN with multiple doses of gadobenate dimeglumine, although considerably less than with gadodiamide.

Our results showed a non-statistically significant trend towards increased signal changes in patients who underwent previous GBCA administration. The suggested amplifying effect of previous administration of gadodiamide on gadolinium deposition observed following gadobenate dimeglumine ( $\mathrm{RC}$
DN/MCP) has not been previously reported. The mechanism by which prior gadodiamide deposition may potentiate gadolinium deposition following gadobenate dimeglumine is as yet unknown.

A saturation effect was recently reported by Robert et al. [10], who described a curve plateau near the 16 th gadodiamide administration in rats. Our findings do not corroborate this saturation effect since we did not find a plateau of the RC DN/MCP in patients with previous administration of gadodiamide but instead we found a non-statistically significant trend towards an increase in RC DN/MCP in those patients. This may suggest either that saturation does not occur in humans or that it occurs at a similar number of multiple GBCA administrations. Animal studies are not strictly
Fig. 3 Unenhanced axial fast spin-echo T1-weighted MR images at the level of the dentate nucleus on baseline MRI $(\mathbf{a}, \mathbf{c})$ and after 4 administrations of gadobenate dimeglumine $(\mathbf{b}, \mathbf{d})$ in a patient with previous administration of gadodiamide (a, b) and in a patient without known previous administration of other GBCAs (c, d)
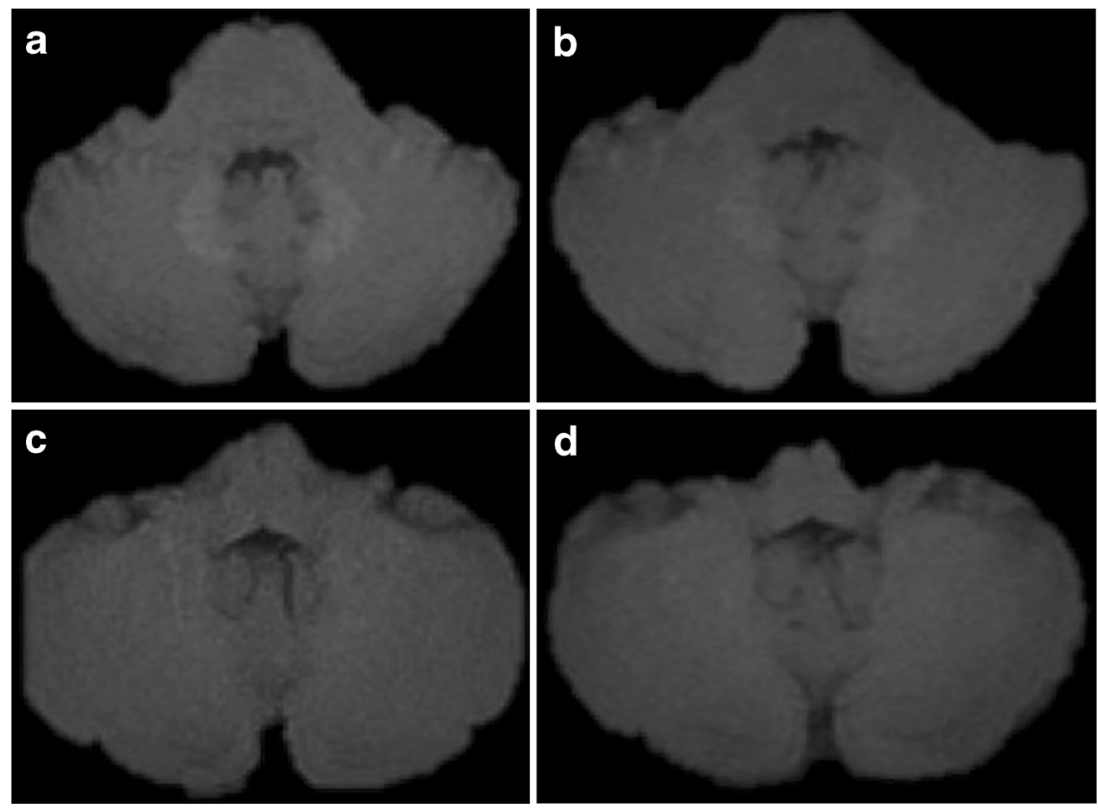
Fig. 4 Box plot representation of a DN/MCP for baseline examinations (Exam Baseline$\left._{\text {e }}\right)$ and last known examinations $\left(\right.$ Exam $\left._{\text {Final }}\right)$ in group 1 (patients with previous exposure to gadodiamide) and group 2 (patients without know previous exposure to gadodiamide) and $\mathbf{b}$ RC DN/MCP signal intensity in the same groups. $D N / M C P$ dentate nucleus-to-middle cerebellar peduncle ratio, $R C$ relative change a

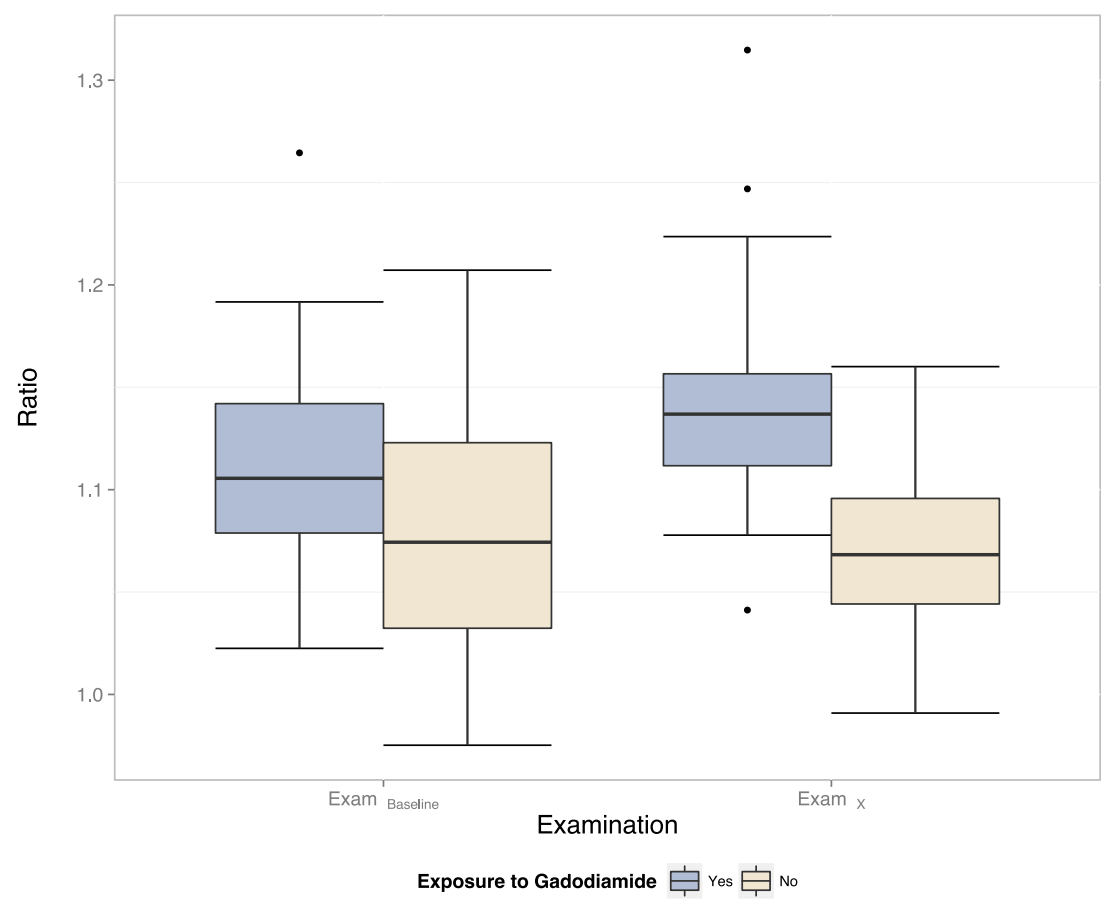

b

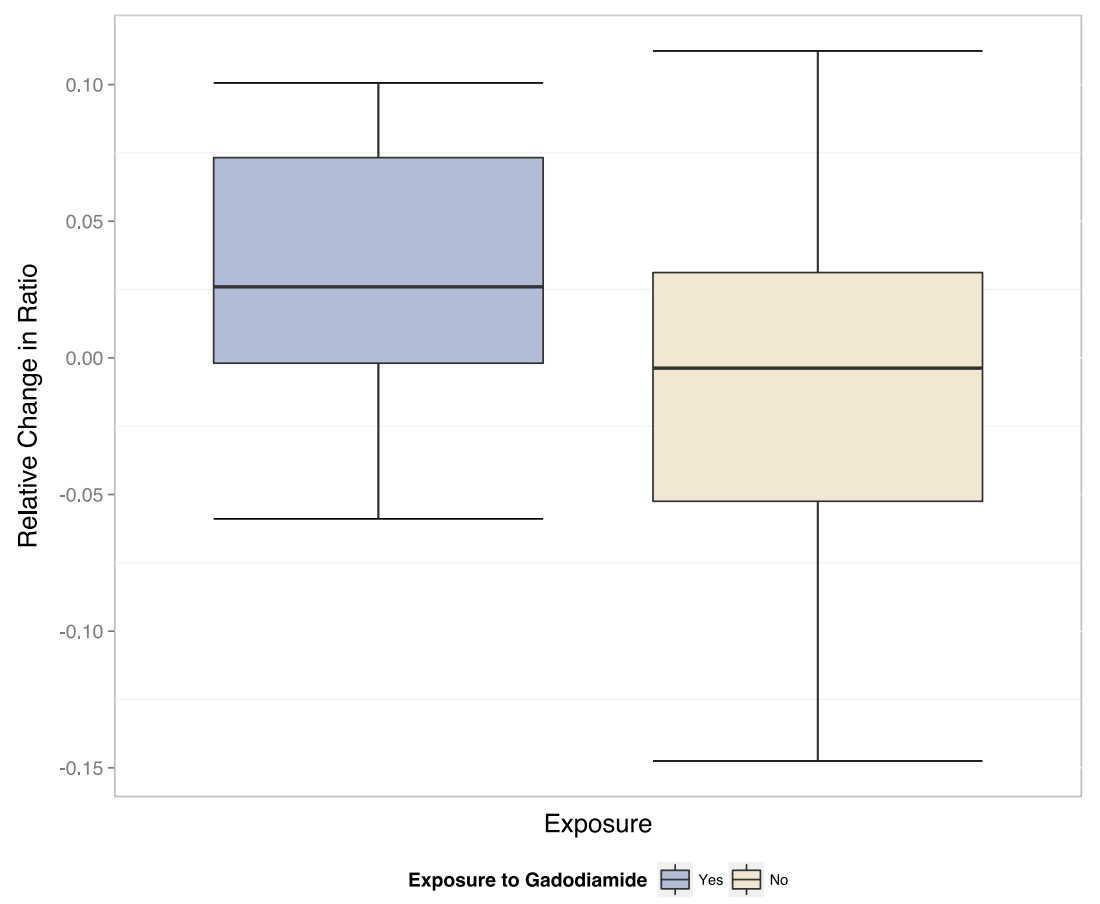

equivalent to human studies; for example, the excretion halflife of GBCAs in healthy rats [20] is markedly shorter than in humans [21], thereby reducing tissue exposure [10] which may suggest that saturation effects may be observed in humans with lesser numbers of GBCA administrations.
However, since none of our patients underwent 16 studies with gadodiamide, we were not able to confirm this finding.

Another observation is that no substantial gadolinium washout occurred in our population during the interval between the last injection of gadodiamide and the last injection 
Fig. 5 Scatter plot representation of $\mathbf{a} \mathrm{DN} / \mathrm{MCP}$ and $\mathbf{b} \mathrm{RC} \mathrm{DN} /$

MCP showing the relationship between the baseline and last MRI examination for patients who received different numbers of doses of gadobenate dimeglumine (MultiHance $®)$ in group 1 (patients with exposure to gadodiamide) and group 2 (patients without known exposure to gadodiamide). The relationship is fitted using GAM as a smoothing function and the shaded areas represent the standard errors. $D N / M C P$ dentate nucleus-to-middle cerebellar peduncle ratio, $R C$ relative change, GAM generalized additive model a

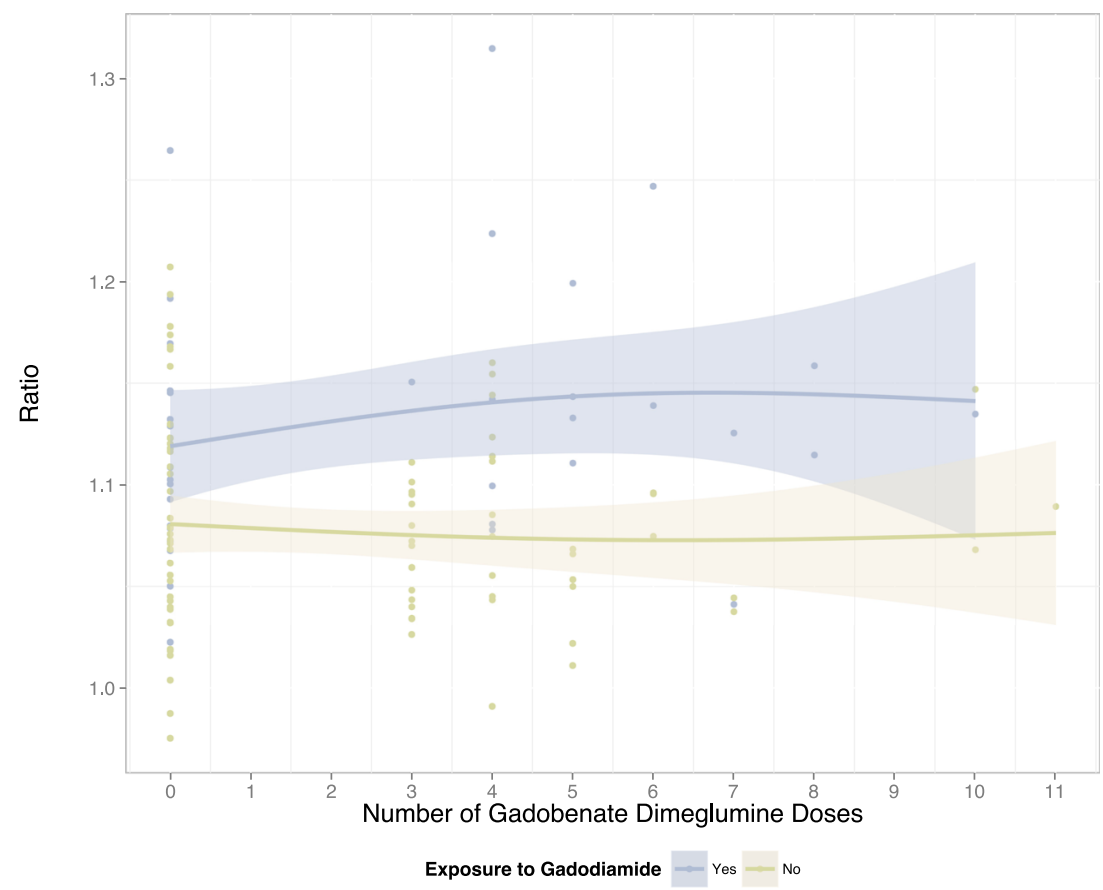

b

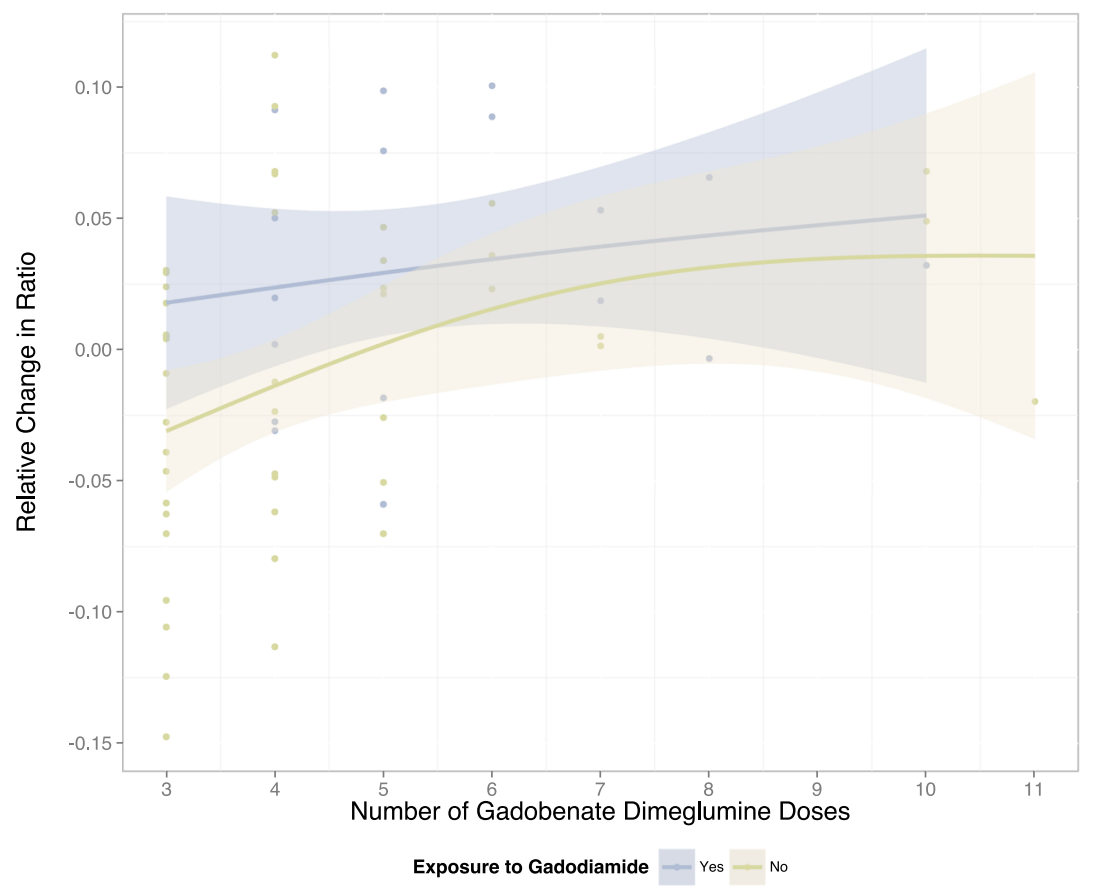

of gadobenate dimeglumine as no increase of RC DN/MCP would be expected if washout had occurred.

Our study had some limitations including the retrospective design and a relatively small sample size. The sample size was possibly the reason for the non-statistically significant trend in RC DN/MCP. Another limitation was the potential confounding effect of variations between the two groups including age, sex, intervals between the examinations, and underlying disease processes. However, we attempted to account for their effects by including these variables in our statistical GAM models. Evaluation of washout over a period of years would require years of not having contrast-enhanced MRI 
studies, something that did not occur in our patients. It is also impossible to exclude that our patients received other GBCAs prior to the first study performed at our institution. We think that this is unlikely as we carefully searched all medical records, and patients who had undergone studies with contrast administration outside our institution were excluded. We also used the differences between the first and last examinations performed with gadobenate dimeglumine to calculate the changes in signal over time, avoiding possible effects of preexisting GBCA administrations.

In conclusion, our results suggest that there is increased $\mathrm{T} 1$ signal change over time in patients who underwent gadobenate dimeglumine-enhanced studies and had prior administration of gadodiamide compared to those who received gadobenate dimeglumine alone. This suggests that a potentiating effect from prior gadodiamide on the subsequent administered more stable gadobenate dimeglumine may occur.

Acknowledgments The scientific guarantor of this publication is Richard C. Semelka. The authors of this manuscript declare no relationships with any companies whose products or services may be related to the subject matter of the article. The authors state that this work has not received any funding. One of the authors has significant statistical expertise. Institutional review board approval was obtained. Written informed consent was waived by the institutional review board. No study subjects or cohorts have been previously reported. Methodology: retrospective, case-control study, performed at one institution.

\section{References}

1. Kanda T, Ishii K, Kawaguchi H, Kitajima K, Takenaka D (2014) High signal intensity in the dentate nucleus and globus pallidus on unenhanced T1-weighted MR images: relationship with increasing cumulative dose of a gadolinium-based contrast material. Radiology 270(3):834-841

2. Errante Y, Cirimele V, Mallio CA, Di Lazzaro V, Zobel BB, Quattrocchi CC (2014) Progressive increase of T1 signal intensity of the dentate nucleus on unenhanced magnetic resonance images is associated with cumulative doses of intravenously administered gadodiamide in patients with normal renal function, suggesting dechelation. Invest Radiol 49(10):685-690

3. Quattrocchi CC, Mallio CA, Errante Y et al (2015) Gadodiamide and dentate nucleus $\mathrm{T} 1$ hyperintensity in patients with meningioma evaluated by multiple follow-up contrast-enhanced magnetic resonance examinations with no systemic interval therapy. Invest Radiol 50(7):470-472

4. Ramalho J, Castillo M, AlObaidy M et al (2015) High signal intensity in globus pallidus and dentate nucleus on unenhanced T1weighted MR images: evaluation of two linear gadolinium-based contrast agents. Radiology 276(3):836-844
5. Kanda T, Osawa M, Oba $\mathrm{H}$ et al (2015) High signal intensity in dentate nucleus on unenhanced T1-weighted MR images: association with linear versus macrocyclic gadolinium chelate administration. Radiology 275(3):803-809

6. Radbruch A, Weberling LD, Kieslich PJ et al (2015) Gadolinium retention in the dentate nucleus and globus pallidus is dependent on the class of contrast agent. Radiology 275(3):783-791

7. Cao Y, Huang DQ, Shih G, Prince MR (2016) Signal change in the dentate nucleus on T1-weighted MR images after multiple administrations of gadopentetate dimeglumine versus gadobutrol. Am J Roentgenol 206(2):414-419

8. McDonald RJ, McDonald JS, Kallmes DF et al (2015) Intracranial gadolinium deposition after contrast-enhanced MR imaging. Radiology 275(3):772-782

9. Kanda T, Fukusato T, Matsuda M et al (2015) Gadolinium-based contrast agent accumulates in the brain even in subjects without severe renal dysfunction: evaluation of autopsy brain specimens with inductively coupled plasma mass spectroscopy. Radiology 276(1):228-232

10. Robert P, Lehericy S, Grand S et al (2015) T1-weighted hypersignal in the deep cerebellar nuclei after repeated administrations of gadolinium-based contrast agents in healthy rats: difference between linear and macrocyclic agents. Invest Radiol 50(8):473-480

11. Robert P, Violas X, Grand S et al (2016) Linear gadolinium-based contrast agents are associated with brain gadolinium retention in healthy rats. Invest Radiol 51(2):73-82

12. Radbruch A, Weberling LD, Kieslich PJ et al (2015) High-signal intensity in the dentate nucleus and globus pallidus on unenhanced T1-weighted images. Invest Radiol 50(12):805-810

13. Weberling LD, Kieslich PJ, Kickingereder $P$ et al (2015) Increased signal intensity in the dentate nucleus on unenhanced T1-weighted images after gadobenate dimeglumine administration. Invest Radiol 50(11):743-748

14. Frenzel T, Lengsfeld P, Schirmer H, Hütter J (2008) Stability of gadolinium-based magnetic resonance imaging contrast agents in human serum at 37 degrees C. Invest Radiol 43(12):817-828

15. Kasahara S, Miki Y, Kanagaki M et al (2011) Hyperintense dentate nucleus on unenhanced T1-weighted MR images is associated with a history of brain irradiation. Radiology 258(1):222-228

16. Roccatagliata L, Vuolo L, Bonzano L, Pichiecchio A, Mancardi GL (2009) Multiple sclerosis: hyperintense dentate nucleus on unenhanced T1-weighted MR images is associated with the secondary progressive subtype 1 . Radiology 251(2):503-510

17. Core Team R (2015) R: a language and environment for statistical computing. R Foundation for Statistical Computing, Vienna, Austria

18. Hastie TJ, Tibshirani RJ (1990) Generalized additive models, 1st edn. Chapman \& Hall/CRC, London

19. Lin LI (1989) A concordance correlation coefficient to evaluate reproducibility. Biometrics 45(1):255-268

20. Oksendal AN, Hals PA (1993) Biodistribution and toxicity of MR imaging contrast media. J Magn Reson Imaging 1;3(1):157-165

21. Idée J-M, Port M, Raynal I, Schaefer M, Le Greneur S, Corot C (2006) Clinical and biological consequences of transmetallation induced by contrast agents for magnetic resonance imaging: a review. Fundam Clin Pharmacol 20(6):563-576 